\title{
Mesenchymal stem cells protect NOD mice from diabetes by inducing regulatory $T$ cells
}

\author{
A. M. Madec • R. Mallone • G. Afonso • E. Abou Mrad • \\ A. Mesnier • A. Eljaafari • C. Thivolet
}

Received: 26 January 2009/Accepted: 25 March 2009/Published online: 7 May 2009

(C) Springer-Verlag 2009

\begin{abstract}
Aims/hypothesis Displaying immunomodulatory capacities, mesenchymal stem cells (MSCs) are considered as beneficial agents for autoimmune diseases. The aim of this study was to examine the ability of MSCs to prevent autoimmune diabetes in the NOD mouse model.

Methods Prevention of spontaneous insulitis or of diabetes was evaluated after a single i.v. injection of MSCs in 4-week-old female NOD mice, or following the coinjection of MSCs and diabetogenic T cells in irradiated male NOD recipients, respectively. The frequency of
\end{abstract}

Electronic supplementary material The online version of this article (doi:10.1007/s00125-009-1374-z) contains supplementary material, which is available to authorised users.

A. M. Madec $(\triangle) \cdot$ E. Abou Mrad - A. Mesnier $\cdot$ C. Thivolet INSERM U870/INRA 1235, Faculté de Médecine Lyon-Sud, 165 chemin du Grand-Revoyet,

69600 Oullins, France

e-mail: madec@laennec.univ-lyon1.fr

A. M. Madec $\cdot$ E. Abou Mrad $\cdot$ A. Mesnier $\cdot$ C. Thivolet

INSA-Lyon,

Lyon, France

A. M. Madec · E. Abou Mrad - A. Mesnier $\cdot$ C. Thivolet Université Lyon1,

Lyon, France

A. M. Madec $\cdot$ E. Abou Mrad $\cdot$ A. Mesnier $\cdot$ C. Thivolet Hospices Civils de Lyon,

Lyon, France

R. Mallone $\cdot$ G. Afonso

INSERM U561, Hôpital Cochin St Vincent de Paul,

Paris, France

A. Eljaafari

Unité mixte HCL-Biomérieux,

Lyon, France
$\mathrm{CD}^{+} \mathrm{FOXP}^{+}$cells and Foxp 3 mRNA levels in the spleen of male NOD recipients were also quantified. In vivo cell homing was assessed by monitoring 5,6-carboxyfluorescein diacetate succinimidyl ester (CFSE)-labelled $\mathrm{T}$ cells or MSCs. In vitro, cell proliferation and cytokine production were assessed by adding graded doses of irradiated MSCs to insulin B9-23 peptide-specific $T$ cell lines in the presence of irradiated splenocytes pulsed with the peptide. Results MSCs reduced the capacity of diabetogenic T cells to infiltrate pancreatic islets and to transfer diabetes. This protective effect was not associated with the modification of diabetogenic T cell homing, but correlated with a preferential migration of MSCs to pancreatic lymph nodes. While injection of diabetogenic $\mathrm{T}$ cells resulted in a decrease in levels of $\mathrm{FOXP}^{+}$regulatory $\mathrm{T}$ cells, this decrease was inhibited by MSC co-transfer. Moreover, MSCs were able to suppress both allogeneic and insulin-specific proliferative responses in vitro. This suppressive effect was associated with the induction of IL10-secreting $\mathrm{FOXP}^{+} \mathrm{T}$ cells.

Conclusions/interpretation MSCs prevent autoimmune beta cell destruction and subsequent diabetes by inducing regulatory T cells. MSCs may thus offer a novel cell-based approach for the prevention of autoimmune diabetes and for islet cell transplantation.

Keywords Cell therapy $\cdot$ Diabetes $\cdot$ Protection

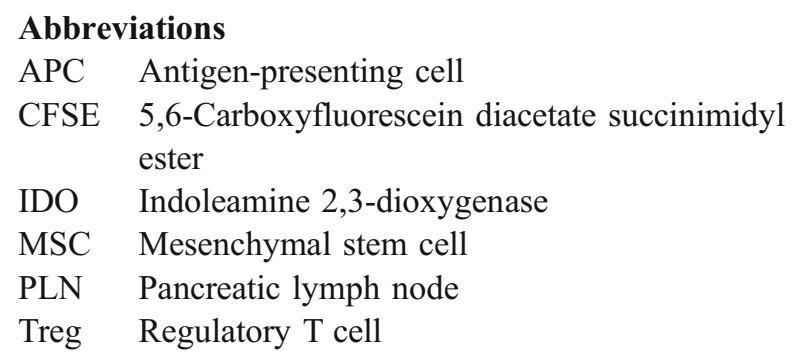




\section{Introduction}

Mesenchymal stem cells (MSCs) are multipotent cells, most of which reside within the bone marrow. These cells, first characterised by Friedenstein and colleagues more than 40 years ago [1], can be induced to undergo differentiation under appropriate conditions into several lineages, including cartilage, bone, adipose and stromal tissues, as reviewed recently $[2,3]$. Studies conducted in both humans and animal models have demonstrated that MSCs are capable of long-term engraftment and multilineage differentiation in vivo.

In addition to these characteristics, which support the successful use of allogeneic MSCs in regenerative therapy, MCSs are able to modulate $\mathrm{T}$ cell responses and/or induce an immunosuppressive local milieu, which make them attractive potential therapeutic agents for various immunological disorders [4]. The i.v. administration of MSCs prolonged skin graft survival modestly but significantly [5], to an extent similar to that seen in response to the immunosuppressive agents currently in use. MSCs have also recently been demonstrated to suppress several $\mathrm{T}$ lymphocyte activities [6] and have been proposed as a possible treatment for acute graft-versus-host disease in humans [7]. Although the mechanisms mediating such effects are only partially understood, it is likely that both cell-to-cell contact and soluble factors are involved [8]. Another property unique to MSCs - a lack of expression of MHC class II and other surface markers such as CD11b, $\mathrm{CD} 14, \mathrm{CD} 31, \mathrm{CD} 34$ or CD45-results in their failure to initiate $\mathrm{CD}^{+} \mathrm{T}$ cell activation and likely explains how MSCs escape the normal process of alloantigen recognition.

Modulation of autoimmunity is therefore considered a potential novel target for MSC-based treatments. It has recently been reported that MSCs are capable of improving experimental autoimmune encephalomyelitis, the murine counterpart of human multiple sclerosis $[9,10]$. In a murine model of rheumatoid arthritis, infusion of MSCs had no beneficial effects on collagen-induced arthritis [11], while another report demonstrated that allogeneic murine MSCs are able to prevent joint tissue damage in rheumatoid arthritis [12]. However, the therapeutic potential of MSCs in type 1 diabetes remains completely unexplored, as reviewed recently by Abdi et al. [13]. The NOD mouse is the foremost model used to assess preventive strategies in type 1 diabetes, as it develops a spontaneous form of autoimmune diabetes that closely resembles the human disease $[14,15]$. Beta cell destruction results from progressive islet infiltration by autoreactive $\mathrm{T}$ cells and macrophages on a permissive genetic background. In this paper we report on the capacity of MSCs to inhibit autoimmune beta cell destruction in the NOD mouse by induction of regulatory $\mathrm{T}$ cells.

\section{Methods}

Isolation, culture and characterisation of mouse MSCs MSCs generated from BALB.B $\left(\mathrm{H}_{2}{ }^{\mathrm{b}}\right)$ mice were a generous gift of E. Simpson (Department of Immunology and Transplantation Biology, Hammersmith Hospital, London, UK) and were cultured as previously described [8]. Before experimental use, the ability of the MSCs to differentiate into adipocytes was tested by adding $10^{-8} \mathrm{~mol} / \mathrm{l}$ dexamethasone to the culture medium for 1 week. Oil Red O dye was used to identify adipocytes. The phenotypic properties of MSCs were also determined on the basis of CD105, CD73, CD29 and CD44 expression and the absence of any hematopoietic (i.e. CD45, CD14, CD11c) or endothelial (i.e. CD31) markers. All antibodies for these experiments were purchased from BD Biosciences Pharmingen (Le Pont-de-Claix, France). MSCs were maintained in culture for less than 20 passages in mouse Mesencult stem cell medium (StemCell Technologies, Meylan, France) before being used.

Mice NOD ( $\left.\mathrm{H}^{\mathrm{g} 7}\right)$ mice (Taconic Europe, Laven, Denmark) were purchased and bred under specific pathogen-free conditions in our animal facility. Spontaneous diabetes starts at 12 weeks of age in females, and the incidence of diabetes in our colony reaches $76 \%$ in females and $15 \%$ in males by 30 weeks of age. Diabetes was assessed by measuring urine glucose with chemstrips (Bayer Diagnostics, Puteaux, France). C57BL/6 (H2 ${ }^{\mathrm{b}}$, MSC-matched) mice were purchased from Charles River (L'Arbresle, France). The institutional review committee for animal experiments approved all the procedures for mouse care and animal killing.

Effects of MSCs on spontaneous and accelerated diabetes To examine the protective effects of MSCs against spontaneous diabetes, a single injection of $10^{5}$ MSCs was administered either i.p. or i.v. to 4-week-old NOD female mice; shaminjected mice served as controls. Accelerated diabetes experiments were performed as previously described, using the adult adoptive cell transfer model $[15,16]$. Briefly, splenic $\mathrm{CD}^{+} \mathrm{T}$ cells from diabetic NOD female mice were purified using enrichment columns (R\&D Systems Europe, Lille, France) and $5 \times 10^{6} \mathrm{~T}$ cells from diabetic mice were co-injected with $10^{3}$ to $10^{6}$ MSCs i.v. into 8- to 12-week-old irradiated (7.5 Gy) male NOD mice. Control mice were injected with T cells alone. At the end of each experiment, the levels of insulitis of five non-diabetic mice from each group were evaluated, as previously described $[15,16]$, using $5 \mu \mathrm{m}$ frozen sections of pancreas as follows: 0 , no infiltration; 1 , peri-islet infiltration; 2, intra-islet infiltration $<50 \%$; 3 , extensive infiltration ( $>50 \%)$. After scoring, a mean was calculated.

Flow cytometry analysis Single cell suspensions were obtained from the spleen and pancreatic lymph nodes 
(PLNs) of each treated animal as previously described [16]. $\mathrm{CD}^{+}$and $\mathrm{CD}^{+}$cells were identified in the different experimental groups using appropriate fluorescent conjugated antibodies. To study cellular homing in vivo, either $\mathrm{T}$ cells or MSCs $\left(10^{7}\right.$ cells $\left./ \mathrm{ml}\right)$ were labelled for $10 \mathrm{~min}$ at $37^{\circ} \mathrm{C}$ with $10 \mu \mathrm{mol} / 1$ 5,6-carboxyfluorescein diacetate succinimidyl ester (CFSE; Molecular Probes, Eugene, OR, USA) immediately before injection, as previously described [16]. CFSEpositive $\mathrm{T}$ cells in the thymus, spleen and PLNs at 3, 5 and 7 days after transfer were analysed by flow cytometry. The percentage of labelled cells was evaluated 1, 3,5 and 7 days after cell transfer. At least $5 \times 10^{5}$ cells were analysed for each sample.

Mononuclear cell culture and proliferation assays To examine the suppressive capacities of MSCs in vitro, we set up a series of allogeneic mixed lymphocyte cultures. MNCs for proliferation assays were obtained from the spleen of either C57BL/6 $\left(\mathrm{H} 2^{\mathrm{b}}\right)$ mice or NOD $\left(\mathrm{H} 2^{\mathrm{g} 7}\right)$ mice. Cells were proliferated in round-bottomed 96 well plates (Dominique Dutscher, Brumath, France) in a total volume of $0.2 \mathrm{ml} \mathrm{RPMI}$ 1640 supplemented with $2 \mathrm{mmol} / \mathrm{l}$ glutamine, $100 \mathrm{U} / \mathrm{ml}$ penicillin, $100 \mu \mathrm{g} / \mathrm{ml}$ streptomycin and 10\% (vol./vol.) heatinactivated fetal calf serum (Invitrogen, Cergy-Pontoise, France). Spleen cells $\left(2 \times 10^{5} /\right.$ well $)$ from NOD mice $\left(\mathrm{H} 2^{\mathrm{g} 7}\right)$ were used as responder cells and primed with irradiated $\mathrm{C} 57 /$ BL6 $\left(\mathrm{H} 2^{\mathrm{b}}\right)$ spleen cells $\left(5 \times 10^{5} /\right.$ well $)$. Cells harvested at day 7 from these primary cultures were then seeded at a concentration of $2 \times 10^{5}$ well together with increasing numbers $\left(10^{2}, 10^{3}, 10^{4}\right.$ per well) of irradiated (60 Gy) MSCs $\left(\mathrm{H}_{2}{ }^{\mathrm{b}}\right)$ and irradiated C57/BL6 splenocytes $\left(2 \times 10^{5} /\right.$ well $)$ as antigenpresenting cells (APCs). In some experiments, $100 \mathrm{U} / \mathrm{ml} \mathrm{IL-2}$ or $100 \mathrm{U} / \mathrm{ml} \mathrm{IFN}-\gamma$ was added to cell cultures to test whether suppression corresponded with $\mathrm{T}$ cell anergy or defects in MHC Class II expression. Under each set of conditions, viability was assessed by the Trypan Blue exclusion test. A total of $0.0185 \mathrm{MBq}(0.5 \mu \mathrm{Ci})\left[{ }^{3} \mathrm{H}\right]$ thymidine (PerkinElmer, Courtaboeuf, France) was added to each well 3 days later, and cells were harvested onto glass fibre Filtermat filters (Skatron Instruments, Lier, Norway) using a 96 well cell harvester (Titertek, Skatron Instruments) after an additional 24 h. A Packard Tricarb beta counter (Perkin-Elmer) was used to measure $\left[{ }^{3} \mathrm{H}\right]$ thymidine uptake. The results were expressed as the mean cpm of sextuplicates (SEs were routinely $<10 \%$ ).

To test whether MSCs could induce cell apoptosis, we determined indoleamine 2,3-dioxygenase (IDO) activity by measuring the conversion of tryptophan to kynurenine in culture supernatant fractions. We examined several culture conditions, including MSCs cultured alone, which were treated with IFN- $\gamma$ or untreated, and MSCs co-cultured with MNCs. Tryptophan and kynurenine concentrations were determined by HPLC by H. Faure (Department of
Integrative Biology, Centre hospitalier universitaire Michalon, Grenoble, France).

Effects of MSCs on antigen-specific Tcell proliferation Insulin B9-23-specific T cell lines were generated from male NOD mice. Animals were immunised subcutaneously with $100 \mu \mathrm{g}$ insulin B9-23 peptide (amino acids 9-23 of the B chain; SHLVEALYLVCGERG) [17, 18] in complete Freund's adjuvant, together with $140 \mu \mathrm{g}$ of a helper peptide consisting of amino acids 128-140 of hepatitis B virus core antigen (TPPAYRPPNAPIL), at the base of the tail. After 12-14 days, an i.p. boost was given at half dose in incomplete Freund's adjuvant without helper peptide. After another 12-14 days, mice were killed, then splenocytes were collected and pulsed for $3 \mathrm{~h}$ with $10 \mu \mathrm{mol} / \mathrm{l}$ of the same insulin peptide. Splenocytes were then washed, plated and cultured for 6-7 days with $5 \mathrm{U} / \mathrm{ml}$ recombinant human IL-2. T cell line specificity was tested with an in-house IFN- $\gamma$ enzyme-linked immunoassay in the presence of fresh splenocytes pulsed with either insulin B9-23 peptide or DMSO.

For MSC suppression assays, male NOD splenocytes pulsed with $10 \mu \mathrm{mol} / 1$ insulin B9-23 peptide and irradiated at 35 Gy were used as APCs and plated at a concentration of 150,000 cells/well in 96 well round-bottomed plates. T cell lines were added at a concentration of 75,000 cells/ well, together with different numbers of irradiated MSCs. After $24 \mathrm{~h}, 50 \mu \mathrm{l}$ of the supernatant fractions were removed from each well and concentrations of IL-4, IL-10, IFN- $\gamma$ were measured by a mouse Th1/Th2 ELISA Ready-SETGO! Kit (eBiosciences, Clinisciences, Montrouge, France). After an additional $48 \mathrm{~h},\left[{ }^{3} \mathrm{H}\right]$ thymidine was added overnight and cells from each plate were harvested and counted the following morning.

To study the effects of MSCs on insulin-specific T cell responses, we performed CFSE dilution assays using flow cytometry. An optimal ratio of one MSC to 250 CFSE-labelled T cells was used in these experiments. To study the induction of regulatory $\mathrm{T}$ cells, cultures were set up as described above using CFSE-labelled T cell lines and CD4-depleted irradiated splenocytes, either in the presence or absence of 1B1 anti-IL10 receptor-blocking monoclonal antibody $(10 \mu \mathrm{g} / \mathrm{ml}$; kindly provided by A. Lehuen, INSERM U561, Paris, France). After 96 h, brefeldin A $(5 \mu \mathrm{g} / \mathrm{ml}$; Sigma, St Quentin Fallavier, France) was added overnight, after which time cells were harvested, stained with anti-CD4-PerCP-Cy5.5, anti-IL-10R-PE (both from BD Biosciences Pharmingen) and antiFOXP3-APC (Miltenyi Biotec, Paris, France) monoclonal antibodies and analysed on a BD FACSAria flow cytometer (BD Biosciences).

FOXP3 analysis Spleens were collected from male NOD mice, 5 days after transfer of $5 \times 10^{6} \mathrm{~T}$ cells, either alone or 
with $10^{6}$ MSCs, as well as from untreated mice. Purified T cells were examined by flow cytometry to evaluate CD4 and FOXP3 expression. Total RNA was isolated from another set of $\mathrm{T}$ lymphocytes from the same experimental mice using the Trizol reagent method (Invitrogen). Total RNA $(1 \mu \mathrm{g})$ was reverse-transcribed in a final volume of $20 \mu \mathrm{l}$ containing $100 \mathrm{U}$ Superscript II (Invitrogen), using $0.5 \mu \mathrm{g}$ random hexamer primers and $0.5 \mu \mathrm{g}$ oligo $(\mathrm{dT})_{15}$ primer (Promega, Charbonnières, France), $6.25 \mathrm{mmol} / \mathrm{l} \mathrm{dNTP}$ (Promega), $0.1 \mathrm{~mol} / \mathrm{l}$ dithiothreitol and 5' First-Strand Buffer (Invitrogen). The resultant cDNA was treated with $5000 \mathrm{U} / \mathrm{ml}$ RNAse-H (New England BioLabs/Ozyme, Saint Quentin en Yvelines, France). Real-time RT-PCR using a LightCycler (Roche Diagnostics, Meylan, France) was performed in a final volume of $20 \mu \mathrm{l}$ containing $15 \mu \mathrm{l}$ of reaction buffer from the FastStart DNA Master Plus SYBR Green I kit (Roche Diagnostics), 10.5 pmol of each forward and reverse specific primer (Eurobio, Les Ulis, France) and $5 \mu \mathrm{l}$ of a 60 -fold dilution of the reverse transcription product. Pairs of PCR primers were designed using the Beacon Designer software (http://beaconsoftware.net/, last accessed 8 April 2009), as follows. Foxp3 primer sequences: Forward: 5'-CCCAG GAAAGACAGCAACCTT-3', Reverse: 5'-TTCTCACAAC CAGGCCACTTG-3'. Samples were quantified using the LightCycler relative quantification software (Roche Diagnostics). Specific mRNA levels were expressed as a percentage relative to the level of mouse Hprt1 mRNA.

Statistical analysis Cell transfers were analysed by comparison of survival curves using Wilcoxon's rank-sum test. Score of insulitis, cell proliferation, cytokine analysis and Foxp 3 expression data were compared using the Student's $t$ test for unpaired samples. A $p$ value of less than $5 \%$ was considered statistically significant.

\section{Results}

MSCs protect NOD mice from diabetes MSCs from BALB. $\mathrm{B}\left(\mathrm{H} 2^{\mathrm{b}}\right)$ mice constitutively expressed CD105, CD73, CD29, CD44 and MHC class I molecules, but not CD14, CD34, CD45 (haematopoietic markers), CD31 (endothelial marker) or MHC class II molecules (Electronic supplementary material [ESM] Fig. 1). The same cells exhibited multilineage differentiation potential as assessed by culturing in adipogenic medium (not shown).

To examine the protective effect of MSCs, we first conducted a series of co-transfer experiments that involved the co-injection of $5 \times 10^{6}$ diabetogenic $\mathrm{T}$ cells and an increasing number of MSCs $\left(10^{3}\right.$ to $\left.10^{6}\right)$ into irradiated NOD male mice. As a control, a series of 43 irradiated male NOD mice were only injected with $5 \times 10^{6} \mathrm{~T}$ cells from diabetic mice. The results from five independent experiments

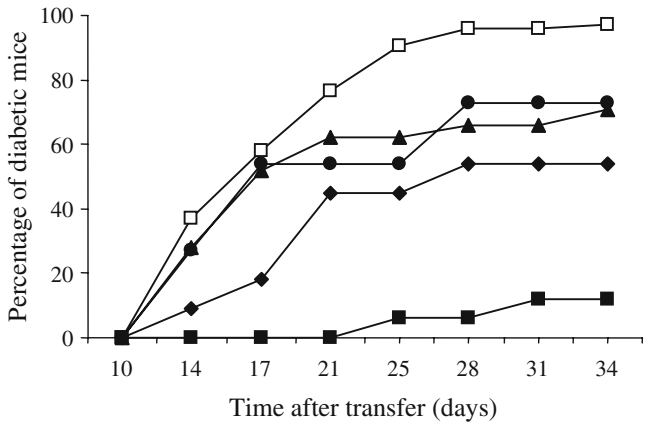

Fig. 1 MSCs confer protection against the transfer of diabetes by NOD splenic T cells. Splenic T cells $\left(5 \times 10^{6}\right)$ from NOD diabetic female mice were intravenously injected into irradiated syngeneic male recipients, either alone (white squares, $n=43$ ) or together with (black symbols) $10^{3}$ (circles, $\left.n=11\right), 10^{4}$ (triangles, $\left.n=21\right), 10^{5}$ (diamonds, $n=11$ ) or $10^{6}$ (squares, $n=15$ ) MSCs. Mice were subsequently followed to determine diabetes development. The results are the means of five independent experiments

are presented in Fig. 1. While the co-injection of $10^{3}$ or $10^{4}$ MSCs together with $5 \times 10^{6}$ diabetogenic $\mathrm{T}$ cells had no significant effect on diabetes prevalence $(9 / 11$ [81.2\%] or $15 /$ $21[71.4 \%]$ vs $42 / 43$ [97.7\%] for mice injected with $5 \times 10^{6}$ diabetogenic T cells alone, $p=\mathrm{NS}$ ), higher concentrations of MSCs $\left(10^{5}\right.$ or $10^{6}$ per mouse) delayed the onset of diabetes and decreased the incidence of diabetes 34 days after cell transfer (6/11 [54.5\%] and 2/16 [12.5\%] diabetic mice, respectively, $p<0.01$ and $p<0.001$ ). As shown in Fig. 2, histological analysis of the pancreases of the mice at 34 days after cell transfer revealed that mice injected with MSCs showed a marked reduction in inflammation compared with those injected with diabetogenic $\mathrm{T}$ cells alone, and that the percentage of intact islets was higher in mice injected with $10^{6}$ MSCs than in those injected with lower levels. The mice injected with diabetogenic $\mathrm{T}$ cells alone had significant insulitis (i.e. stage $2-3$ ) in $90 \%$ of islets. When co-injected intravenously with $5 \times 10^{6}$ diabetogenic T cells into irradiated NOD male mice, splenic T cells $\left(5 \times 10^{6}\right)$ from protected animals did not exert a protective effect (data not shown).

To examine the effect of MSCs on the incidence of spontaneous diabetes, we treated 4-week-old female mice with a single i.p. or i.v. injection of $10^{5}$ MSCs. As shown in Fig. 3, the injection delayed the spontaneous onset of diabetes and decreased the incidence of the disease, with 16 out of $27(59.3 \%)$ MSC-treated mice vs 26 out of 26 $(100 \%)$ control animals becoming diabetic at 36 weeks of age $(p<0.05)$.

Migration of CFSE-labelled cells To examine whether MSCs modify $\mathrm{T}$ cell homing to the pancreas and lymphoid organs, we performed a series of co-transfer experiments using CFSE-labelled, intravenously injected $\mathrm{T}$ cells to follow their migration in irradiated recipients (ESM Fig. 2 and Table 1). Although MSC-injected mice displayed 


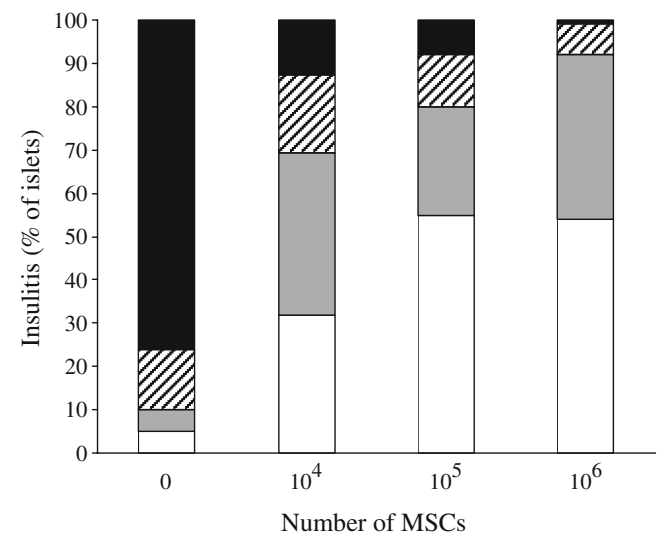

Fig. 2 MSC administration reduces the severity of insulitis. Diabetogenic NOD spleen cells $\left(5 \times 10^{6}\right)$ were intravenously injected into irradiated syngeneic male recipients, either alone or together with $10^{4}$, $10^{5}$ or $10^{6}$ MSCs. Non-diabetic mice were killed 34 days after cell transfer and the insulitis level scored on pancreatic sections. White fill, stage 0 , no infiltration; grey fill, stage 1 , peri-islet infiltration; hatched fill, stage 2, intra-islet infiltration $<50 \%$; black fill, stage 3 , extensive infiltration $(\geq 50 \%)$. A mean score was calculated from five mice per group

tendencies towards a decrease in the number of diabetogenic $\mathrm{T}$ cells in the spleen and an increase in the number in PLNs as compared with mice that received $\mathrm{T}$ cells alone, these differences were not statistically significant. We therefore conclude that the MSCs did not interfere with T cell homing.

Next, we followed the migration of MSCs in recipient mice, using CFSE-labelled i.v. injected MSCs (ESM Fig. 2). As illustrated in Fig. 4, the concentration of MSCs was significantly higher in the PLNs than in the spleen, with peak values achieved at day $5(4.40 \pm 0.72 \%$ vs $1.86 \pm 0.14 \%$, respectively, $p<0.01)$. Interestingly, these differences persisted 18 days later $(1.75 \pm 0.25 \%$ vs $0.52 \pm 0.04 \%$, respectively, $p<0.01)$. However, we were not able to detect any CFSE-labelled MSCs in the islets of recipient mice by direct immunofluorescence analysis of pancreatic sections from recipient mice at 5,11 and 18 days after cell transfer (data not shown).

MSCs inhibit alloreactive NOD T cells in secondary H2mismatched mixed lymphocyte reactions To test their ability to inhibit alloreactivity, MSCs were added to secondary allogeneic mixed lymphocyte reactions. As few as $10^{2}$ MSCs were able to completely suppress alloreactive T cell proliferation (Fig. 5). The addition of exogenous IL-2 to cell cultures did not rescue $\mathrm{T}$ cells from MSC suppression (Fig. 5). Moreover, induction of MHC Class II expression by pre-treating MSCs with IFN- $\gamma$ (ESM Fig. 1) prior to secondary mixed lymphocyte reactions did not result in the recovery of $\mathrm{T}$ cell proliferation (data not shown), which suggests that the defect in MHC class II expression cannot account for MSC suppression.
Furthermore, no IDO activity was detected under any of the mixed lymphocyte reaction co-culture conditions; the kynurenine level was $<0.6 \mathrm{mmol} / \mathrm{l}$ and the tryptophan level was $4.9 \mathrm{mmol} / 1$ in all supernatant fractions, compared with $5.9 \mathrm{mmol} / 1$ and $<0.6 \mathrm{mmol} / \mathrm{l}$, respectively, in the supernatant fractions of positive controls. MSC suppression was not related to the subsequent reduction of cell number and viability measured by the Trypan Blue exclusion method.

MSCs suppress beta cell-specific $T$ cell responses and induce IL-10-producing regulatory T cells in vitro Finally, we tested whether the MSCs could also suppress $T$ cell responses relevant to autoimmune diabetes in vitro. We chose to study responses against the key epitope, insulin B9-23, as it has been shown to play a critical, and likely initiating, role in the autoimmune beta cell destruction [18]. As previously observed for alloreactive T cell responses, NOD insulin B923-specific proliferative responses were significantly suppressed by MSCs in a dose-dependent fashion, from $75 \%$ with $10^{2}$ MSCs to $100 \%$ with $10^{5}$ MSCs (Fig. 6a). As shown in Fig. 6b, this suppression was associated with a dosedependent increase in IL-10 secretion ( $p<0.001$ vs no MSCs).

Insulin-specific T cell responses in the presence of MSCs were further investigated by flow cytometry (Fig. 7). Using CFSE dilution assays, we observed the inhibition of $\mathrm{T}$ cell proliferation in the presence of MSCs $\left(\mathrm{CD}^{+} \mathrm{CFSE}^{+} \mathrm{T}\right.$ cells: $2.7 \%$ vs $0.6 \%$ in the absence and presence of MSCs, respectively) (Fig. 7a), and this effect was associated with the induction of $\mathrm{IL}-10$-producing $\mathrm{FOXP}^{+}$regulatory $\mathrm{T}$ cells (Tregs) (Fig. 7b). Indeed, while some Treg induction was triggered by epitope stimulation alone $(2.7 \%$ vs $6.4 \%$ in the absence and presence of peptide, respectively), the addition of MSCs to the culture significantly increased the proportion of Tregs. This MSC-mediated Treg induction

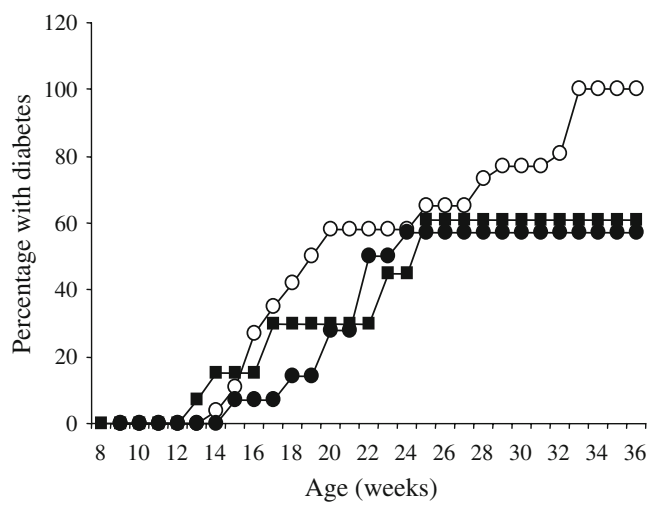

Fig. 3 MSCs protect female NOD mice from spontaneous diabetes. A single injection of $10^{5}$ MSCs was administered either intraperitoneally (black circles, $n=14$ ) or intravenously (squares, $n=13$ ) to 4 -week-old NOD female mice. The control mice were injected with PBS alone (open squares, $n=26$ ). Animals were subsequently followed to determine diabetes development 
Table 1 Effects of MSCs on the migration of $\mathrm{CFSE}^{+} \mathrm{T}$ cells

\begin{tabular}{|c|c|c|c|c|c|c|}
\hline \multirow{2}{*}{$\begin{array}{l}\text { Time after transfer (days) } \\
10^{6} \mathrm{MSCs}\end{array}$} & \multicolumn{2}{|l|}{3} & \multicolumn{2}{|l|}{5} & \multicolumn{2}{|l|}{7} \\
\hline & - & + & - & + & - & + \\
\hline \multicolumn{7}{|c|}{ Percentage of CFSE-labelled T cells } \\
\hline Spleen & $1.79 \pm 0.54$ & $1.46 \pm 0.35$ & $1.58 \pm 0.62$ & $0.88 \pm 0.30$ & $0.79 \pm 0.44$ & $0.20 \pm 0.12$ \\
\hline PLNs & $0.41 \pm 0.14$ & $0.91 \pm 0.52$ & $0.48 \pm 0.02$ & $0.71 \pm 0.23$ & $0.12 \pm 0.08$ & $0.14 \pm 0.03$ \\
\hline Thymus & $0.16 \pm 0.04$ & $0.18 \pm 0.08$ & $0.04 \pm 0.005$ & $0.04 \pm 0.006$ & $0.01 \pm 0.003$ & $0.01 \pm 0.002$ \\
\hline
\end{tabular}

The results are the mean \pm SD of three independent experiments. The percentage of CFSE-labelled T cells was determined by FACS analysis 3,5 and 7 days after the transfer of $5 \times 10^{6} \mathrm{~T}$ cells. No significant difference was seen between the experimental groups

was not antigen specific ( $21.3 \%$ vs $20.2 \%$ in the absence and presence of the insulin B9-23 epitope, respectively). Thus, these results indicate that MSCs are able to promote the generation or expansion of Tregs producing both IL-10 and FOXP3. Importantly, however, MSC-mediated suppression was not IL-10-dependent, as blockade of the IL-10 receptor with a neutralising antibody did not prevent the inhibition of $\mathrm{T}$ cell proliferation $(2.3 \%$ vs $0.5 \%$ in the absence and presence of MSCs, respectively) (Fig. 7c).

Tregs are induced in vivo following MSC administration To explore the possibility that the in vivo immunosuppressive action of MSCs was mediated by activation of Tregs in vivo, we analysed the frequency of $\mathrm{CD} 4^{+} \mathrm{FOXP} 3^{+}$cells in the spleen of irradiated male NOD mice 5 days after i.v. cell transfer. In mice, at day 5 , the frequency of $\mathrm{CD} 4^{+} \mathrm{FOXP} 3^{+}$ lymphocytes in the frequency in mice treated with diabetogenic $\mathrm{T}$ cells alone $(n=5)$ was lower than the frequency in untreated mice $(2.9 \pm 0.3 \%$ vs $4.9 \pm 1.2 \%, p<0.01)$, while the

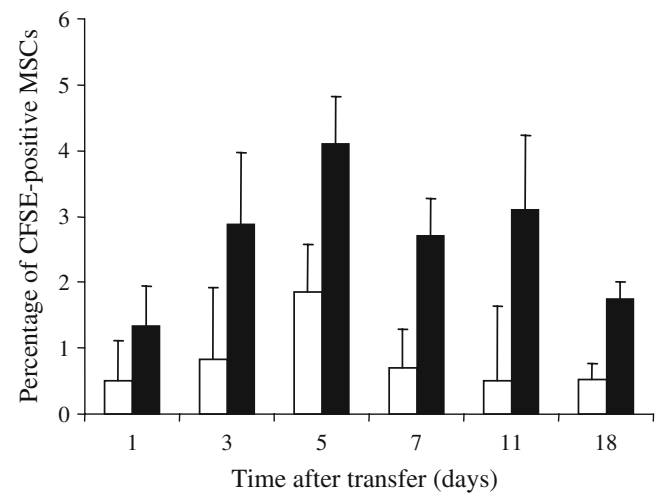

Fig. 4 Injected MSCs preferentially home to PLNs. CFSE-labelled MSCs were co-transferred with $5 \times 10^{6}$ diabetogenic $\mathrm{T}$ cells into irradiated male NOD recipients. Mice were subsequently killed at the indicated time points, and the number of $\mathrm{CFSE}^{+} \mathrm{MSCs}$ in the spleen (white bars) and PLNs (black bars) were determined by flow cytometry. The graph shows the percentage of $\mathrm{CFSE}^{+} \mathrm{MSCs}$ injected that were detected in the spleen and PLNs. All values are the mean \pm $\mathrm{SD}$ of measurements made in triplicate co-transfer of MSCs with diabetogenic T cells resulted in the recovery of the proportion of $\mathrm{CD}^{+} \mathrm{FOXP}^{+}$cells (a representative experiment is shown on Fig. 8). Foxp3 mRNA was also expressed at significantly higher levels in T lymphocytes isolated from the splenocytes of both untreated NOD mice $(n=5)$ and mice receiving a mixture of diabetogenic T cell and MSCs $(n=7)$, compared with the mice that received diabetogenic T cells alone $(n=5)(p<0.001)$ (Fig. 9). We also analysed the frequency of $\mathrm{CD} 4^{+} \mathrm{FOXP} 3^{+}$cells in the PLNs of recipient animals. At day 5, the frequency of $\mathrm{CD} 4{ }^{+} \mathrm{FOXP} 3^{+}$lymphocytes was lower in mice treated with diabetogenic $\mathrm{T}$ cells alone $(n=3)$ than in untreated mice $(p<0.01 ; n=3)$, but co-transfer of MSCs with diabetogenic T cells resulted in the recovery of the percentage of $\mathrm{CD}^{+}{ }^{+} \mathrm{FOXP}^{+}$cells in the PLNs $(7.6 \pm 0.8 \% ; n=3)$.

\section{Discussion}

Although the immunosuppressive capacity of MSCs has been demonstrated in several medical conditions, few

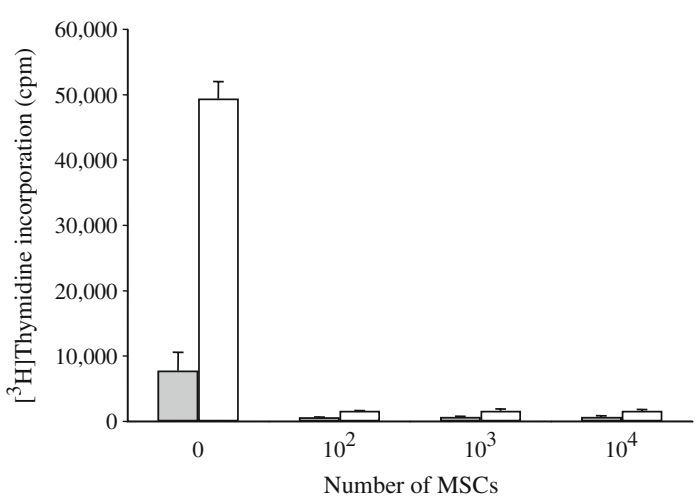

Fig. 5 MSCs suppress the proliferation of alloreactive NOD T cells. Increasing numbers of MSCs were added to secondary allogeneic mixed lymphocyte reactions. The graph shows $\left[{ }^{3} \mathrm{H}\right]$ thymidine incorporation over $25 \mathrm{~h}$. Experiments were performed either in the absence (grey bars) or the presence (open bars) of IL-2. The results are the mean $\pm \mathrm{SD}$ of three independent experiments 

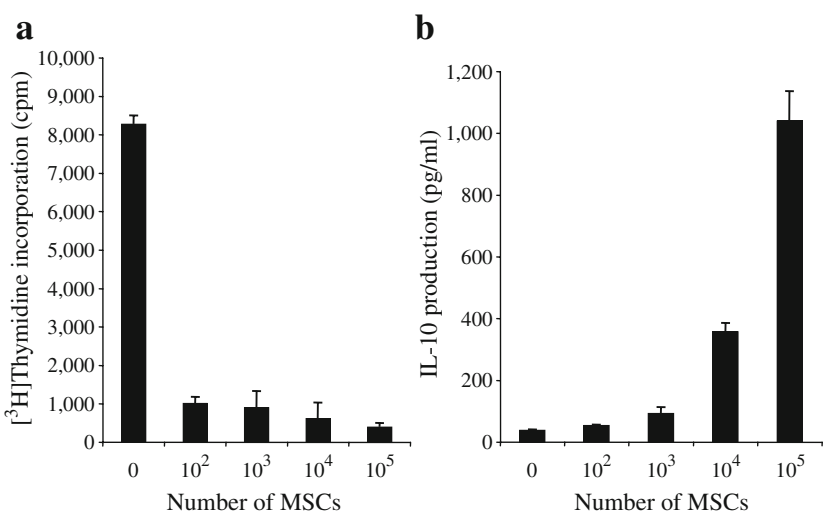

Fig. 6 MSCs suppress insulin-specific T cell responses in vitro. a Insulin B9-23-specific $\mathrm{T}$ cells were stimulated with B9-23-pulsed irradiated splenocytes in the absence or presence of MSCs. $\left.{ }^{3} \mathrm{H}\right]$ Thymidine incorporation after a culture period of $72 \mathrm{~h}$ is shown. Basal proliferation (i.e. on control-pulsed APCs) was $<500 \mathrm{cpm}$. b The IL-10 level in $24 \mathrm{~h}$ culture supernatant fractions was measured by ELISA. All values are expressed as the mean \pm SD of measurements made in triplicate. The results in (a) and (b) are representative of three independent experiments. Irradiated splenocyte APCs alone did not display any proliferation or IL-10 secretion (data not shown)

studies have evaluated their capacity to inhibit $\mathrm{T}$ cell function in autoimmune diseases. MSCs exhibit the intriguing characteristics of being able to escape immune recognition and to inhibit immune responses. In mixed lymphocyte reactions, MSCs suppress T cell proliferation independently of MHC matching between $\mathrm{T}$ cells and MSCs [19, 20]. Murine MSCs have been shown to prevent experimental autoimmune encephalomyelitis in mice through the induction of peripheral tolerance against the pathogenic antigen $[9,10]$. This protection was effective when the MSCs were administered at the onset of the disease or at the peak of the disease, but not after disease stabilisation. Controversial results were obtained in collagen-induced arthritis, but recent reports have shown that MSC infusion prevented tissue damage and exerted immunomodulatory effects by educating antigen-specific Tregs $[11,12]$.

In the present study we examined the effects of the administration of a single dose MSCs in NOD mice, a mouse model of human type 1 diabetes. We provide strong evidence that MSCs confer significant protection against both $\mathrm{T}$ cell-transferred and spontaneous disease. This therapeutic effect correlates with decreased islet infiltration, MSC homing to the draining PLNs, and in vitro suppression of both allo- and beta cell-specific responses. The induction of FOXP3 ${ }^{+} \mathrm{CD}^{+}$Tregs secreting IL-10 was also observed in vitro, although IL-10 did not prove to be involved in the suppressive mechanisms. Despite this apparent discrepancy, our results are in line with those of Thornton and Shevach [21] for mouse Tregs, and of Jonuleit et al. [22] for human Tregs. In both models, Tregs were induced and produced both IL-10 and TGF $\beta$, but their suppressive activity was not dependent on either cytokine. Our results are also in line with other studies suggesting that MSC-mediated suppression may be contact-dependent in vitro but cytokinemediated in vivo [4, 21, 22]. Indeed, this discrepancy between in vitro and in vivo MSC suppression studies is reminiscent of the conundrum regarding the mechanisms of suppression of murine Tregs, which also display distinct behaviours in vitro and in vivo [23]. Our results also highlight important differences between mouse and human MSCs [24, 25]. Glennie et al. [25] reported that human MSC-mediated inhibition induces an unresponsive $\mathrm{T}$ cell profile that can be reverted by the addition of exogenous IL-2. Interestingly, IL-2 did not restore T cell proliferation in our experiments, suggesting that the MSCs did not induce classical $\mathrm{T}$ cell anergy [25]. Moreover, since the number and viability of the cells were not affected, we conclude that

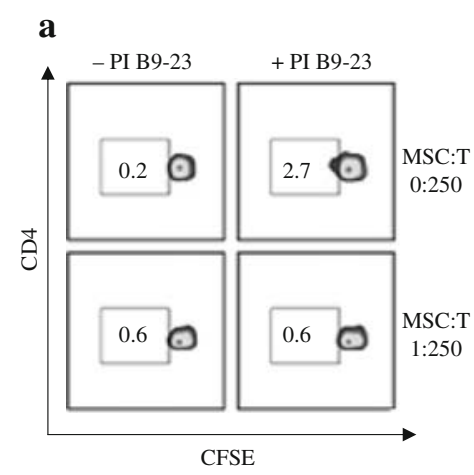

Fig. 7 MSC suppression of insulin-specific $\mathrm{T}$ cell responses is associated with induction of IL-10-producing Tregs. a Insulin B9-23 peptide (PI B9-23)-specific proliferation in the absence (upper panels) or presence (lower panels) of MSCs using CFSE-labelled insulinspecific T cells. Cells were gated on the $\mathrm{CD}^{+} \mathrm{CFSE}^{+}$fraction. $\mathbf{b}$ Induction

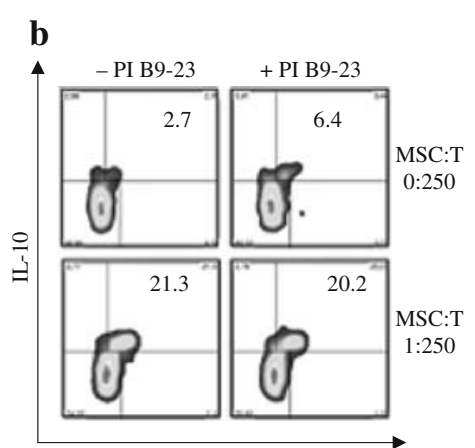

FOXP3

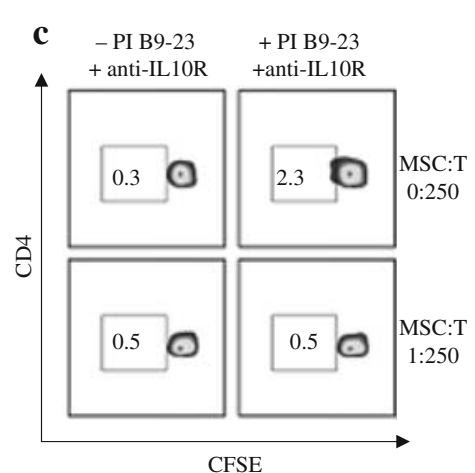

of $\mathrm{IL}-10^{+} \mathrm{FOXP} 3^{+} \mathrm{CD}^{+} \mathrm{T}$ cells. These experiments were conducted under the same conditions as those in panel $\mathbf{a}$, with cells gated on the $\mathrm{CD}^{+} \mathrm{CFSE}^{+}$fraction. c PI B9-23-specific proliferation in the presence of an anti-IL-10 receptor blocking monoclonal antibody 
Fig. 8 MSCs induce Tregs in vivo. The frequency of $\mathrm{CD}^{+}{ }^{+} \mathrm{FOXP}^{+}{ }^{+} \mathrm{T}$ cells isolated from the spleen of control NOD mice (a), mice injected with $\mathrm{T}$ cells from diabetic animals alone (b) or together with MSCs (c). Plots representative of five experiments are shown a

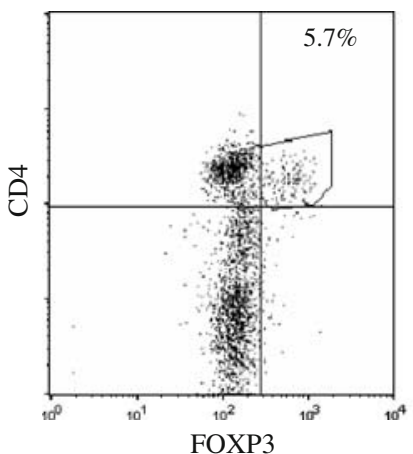

b

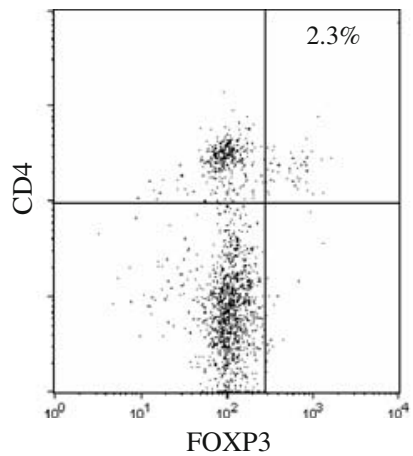

c

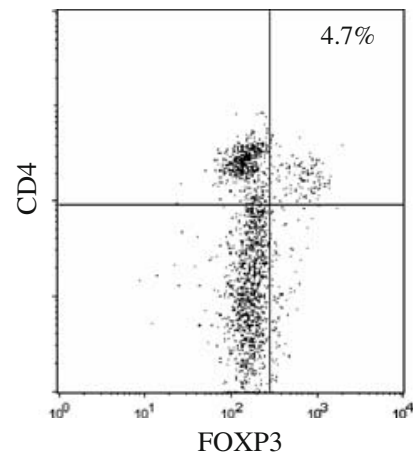

MSCs did not induce T cell apoptosis. One other established mechanism of human MSC-mediated suppression is IDO activity upregulation. IDO activation is induced by IFN- $\gamma$, and IDO catalyses the conversion of tryptophan into kynurenine [26, 27]. Activation of IDO thus causes tryptophan depletion, resulting in reduced lymphocyte proliferation [28]. In contrast to previous findings in human MSCs stimulated by IFN- $\gamma$ [29], we did not detect significant IDO activity in MSC-suppressed mixed lymphocyte reactions, even in the presence of IFN- $\gamma$ (not shown). Thus, the mechanism of MSC-mediated suppression remains controversial. However, although we do not have direct evidence, our data are compatible with a contact-dependent mechanism of suppression, perhaps mediated by an intermediate mediator linking the MSCs to Treg induction. In this respect, Li et al. recently showed that Treg-inducing dendritic cells can be generated through direct cell-to-cell contact between haematopoietic stem cells and MSCs by activation of the Notch

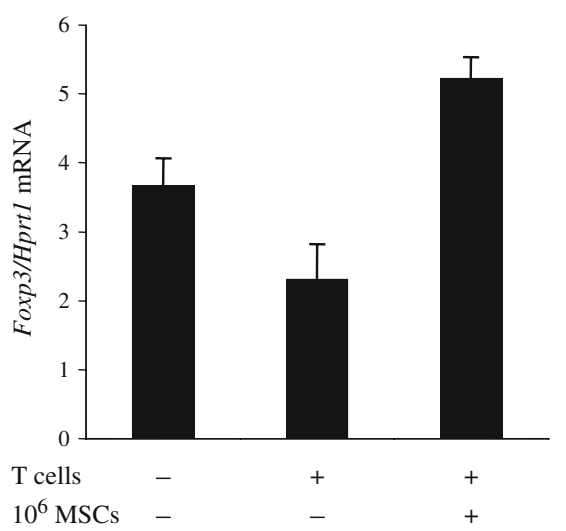

Fig. 9 Foxp3 expression levels in $\mathrm{T}$ splenocytes by real-time PCR. The addition of MSCs to $\mathrm{T}$ lymphocytes from diabetic mice significantly increased the level of Foxp3 expression $(p<0.001)$. Values are the mean \pm SD of relative expression ratio as compared with the Hprt1 reference gene (untreated mice and mice treated with diabetogenic T cells, $n=5$; mice treated with diabetogenic $\mathrm{T}$ cells and MSCs, $n=7$ ) signalling pathway [30]. Whether a similar mechanism is at play in our model requires further investigation.

The administration of a single dose of adult MSCs was sufficient to prevent the onset of diabetes. One possible explanation for this efficacy could be related to the regeneration of pancreatic islets by MSCs; however, we considered this hypothesis unlikely since protection was obtained during the early inflammatory phase of the disease. Moreover, Urban et al. [31] have recently reported in a streptozotocin-induced mouse model of diabetes that MSCs cannot induce pancreas regeneration by themselves, as they require co-injection of bone marrow stem cells. In addition, clinical efficacy in our model was correlated with greatly decreased insulitis scores, further suggesting immune-mediated, rather than regenerative, mechanisms. In a recent paper, Tritt et al. [32] demonstrated that a decrease in the frequency of $\mathrm{CD}^{+}$Tregs contributes to the onset of autoimmune diabetes. We therefore measured the expression of FoxP3 on cells in the spleen and PLNs of MSC-treated or untreated mice and found that MSC treatment increased FOXP3 $\mathrm{T}$ cell frequency. These results correlate with the in vitro findings showing that the immunosuppressive activity of MSCs is correlated with the induction of $\mathrm{FOXP}^{+}{ }^{+}$cells. Following this model of Treg-mediated suppression by MSCs, it was surprising to observe that splenic T cells from protected animals were not able to transfer protection. This discrepancy may be due to the preferential homing of induced Tregs towards PLNs. Indeed, MSCs were found to preferentially home to PLNs rather than the spleen. It is therefore reasonable to hypothesise that MSC-induced Tregs may also preferentially reside in PLNs or in the islets themselves. Supporting this possibility, Tritt et al. [32] reported that Tregs preferentially localise in the pancreatic environment, where they suppress the accumulation and function of effector T cells.

In conclusion, we have demonstrated the therapeutic potential of the administration of a single dose of adult MSCs to prevent the development of type 1 diabetes beta cell autoimmunity. This clinical efficacy correlates with the 
suppressive effects of MSCs on both allogeneic and autoimmune responses in vitro, and with the induction of Tregs both in vivo and in vitro. MSC suppression does not require MHC matching or any additional immunosuppressive therapy. This may have important clinical implications for type 1 diabetes immune interventions.

Acknowledgements We are indebted to E. Simpson (Department of Immunology and Transplantation Biology, Hammersmith Hospital, London, UK) for the gift of the MSCs, to A. Durand (INSERM U870/ INRA 1235/INSA/Université Lyon1/Hospices Civils de Lyon, Lyon, France) for excellent technical assistance and for assistance with the NOD colony, and to J. Plumas (Laboratoire R\&R, EFS Rhône-Alpes, Grenoble, France) for analysis of the IDO results. This study was supported by INSERM grants to A. M. Madec and C. Thivolet, and by grants from the European Foundation for the Study of Diabetes (EFSD-JDRF-Novo Nordisk award), the Fondation Recherche Médicale (Installation Nouvelle Equipe) and the Programme National de Recherche sur le Diabète 2007 (to R. Mallone).

Duality of interest The authors declare that there is no duality of interest associated with this manuscript.

\section{References}

1. Friedenstein AJP, Petrokova KV (1966) Osteogenesis in transplants of bone marrow cells. J Embryol Exp Morphol 16:381-390

2. Barry FP, Murphy JM, English K, Mahon BP (2005) Immunogenicity of adult mesenchymal stem cells: lessons from the fetal allograft. Stem Cells Dev 14:252-265

3. Barry FP, Murphy JM (2004) Mesenchymal stem cells: clinical applications and biological characterization. Int J Biochem Cell Biol 36:568-584

4. Nauta AJ, Fibbe WE (2007) Immunomodulatory properties of mesenchymal stem cell. Blood 15:3499-3506

5. Bartholomew A, Sturgeon C, Siatskas M et al (2002) Mesenchymal stem cells suppress lymphocyte proliferation in vitro and prolong skin graft survival in vivo. Exp Hematol 30:42-48

6. Rasmusson I, Ringden O, Sundberg B, Le Blanc K (2003) Mesenchymal stem cells inhibit the formation of cytotoxic $\mathrm{T}$ lymphocytes, but not activated cytotoxic $\mathrm{T}$ lymphocytes or natural killer cells. Transplantation 76:1208-1213

7. Le Blanc K, Rasmusson I, Sundberg B et al (2004) Treatment of severe acute graft-versus-host disease with third party haploidentical mesenchymal stem cells. Lancet 363:1439-1441

8. Krampera M, Glennie S, Dyson J et al (2003) Bone marrow mesenchymal stem cells inhibit the response of naive and memory antigen-specific T cells to their cognate peptide. Blood 101:3722-3729

9. Zappia E, Casazza S, Pedemonte E et al (2005) Mesenchymal stem cells ameliorate experimental autoimmune encephalomyelitis inducing T cell anergy. Blood 106:1755-1761

10. Zhang J, Li Y, Chen J et al (2005) Human bone marrow stromal treatment improves neurological functional recovery in EAE mice. Exp Neurol 195:16-26

11. Djouad F, Fritz V, Apparailly F et al (2005) Reversal of the immunosuppressive properties of mesenchymal stem cells by tumor necrosis factor $\alpha$ in collagen-induced arthritis. Arthritis Rheum 52:1595-1603

12. Augello A, Tasso R, Negrini SM, Cancedda R, Pennesi G (2007) Cell therapy using allogeneic bone marrow mesenchymal stem cells prevents tissue damage in collagen-induced arthritis. Arthritis Rheum 56:1175-1186
13. Abdi R, Fiorina P, Adra CH, Atkinson M, Sayegh MH (2008) Immunomodulation by mesenchymal stem cells. A potential therapeutic strategy for type 1 diabetes. Diabetes 57:1759-1767

14. Kikutani H, Makino S (1992) The murine autoimmune diabetes model: NOD and related strains. Adv Immunol 51:285-322

15. Thivolet C, Bendelac A, Bedossa P, Bach JF, Carnaud C (1991) $\mathrm{CD}^{+} \mathrm{T}$ cell homing to the pancreas in the nonobese diabetic mouse is $\mathrm{CD}^{+} \mathrm{T}$ cell-dependent. J Immunol 146:85-88

16. Aspord C, Czerkinsky C, Durand A, Stefanutti A, Thivolet C (2002) $\alpha 4$ Integrins and L-selectin differently orchestrate T-cell activity during diabetes prevention following oral administration of CTB-insulin. J Autoimmun 19:223-232

17. Daniel D, Gill RG, Schoot N, Wegmann D (1995) Epitope specificity, cytokine production profile and diabetogenic activity of insulin-specific T cell clones isolated from NOD mice. Eur J Immunol 25:1056-1062

18. Nakayama M, Abiru N, Moriyama H et al (2005) Prime role for an insulin epitope in the development of type 1 diabetes in NOD mice. Nature 435:220-223

19. Di Nicola M, Carlo-Stella C, Magni M et al (2002) Human bone marrow stromal cells suppress T-lymphocyte proliferation induced by cellular or nonspecific mitogenic stimuli. Blood 99:3838-3843

20. Rasmusson I (2006) Immune modulation by mesenchymal stem cells. Exp cell Res 312:2169-2179

21. Thornton AM, Shevach EM (1998) $\mathrm{CD} 4^{+} \mathrm{CD} 25^{+}$immunoregulatory $\mathrm{T}$ cells suppress polyclonal activation in vitro by inhibiting interleukin 2 production. J Exp Med 188:287-296

22. Jonuleit H, Schmitt E, Schuler G, Knop J, Enk AH (2000) Induction of interleukin 10-producing, non-proliferating $\mathrm{CD}^{+} \mathrm{T}$ cells with regulatory properties by repetitive stimulation with allogeneic immature human dendritic cells. J Exp Med 192: $1213-1222$

23. Bach JF (2003) Regulatory $T$ cells under scrutiny. Nat Rev Immunol 3:189-198

24. Klyushenkova E, Mosca JD, Zermetkina V et al (2005) T cell responses to allogeneic human mesenchymal stem cells: immunogenicity, tolerance, and suppression. J Biomed Sci 12:45-57

25. Glennie S, Soeiro I, Dyson PJ, Lam EW, Dazzi F (2005) Bone marrow mesenchymal stem cells induce division arrest anergy of activated T cells. Blood 105:1815-1822

26. Le Rond S, Gonzalez A, Gonzalez AS, Carosella ED, Rouas-Freiss N (2005) Indoleamine 2,3 dioxygenase and human leucocyte antigen-G inhibit the T-cell alloproliferative response through two independent pathways. Immunology 116:297-307

27. Plumas J, Chaperot L, Richard MJ, Molens JP, Bensa JC, Favrot MC (2005) Mesenchymal stem cells induce apoptosis of activated T cells. Leukemia 19:1597-1604

28. Terness P, Bauer TM, Rose L et al (2002) Inhibition of allogeneic $\mathrm{T}$ cell proliferation by indoleamine 2,3-dioxygenase-expressing dendritic cells: mediation of suppression by tryptophan metabolites. J Exp Med 196:447-457

29. Frumento G, Rotondo R, Tonetti M, Ferrara GB (2001) T cell proliferation is blocked by indoleamine 2,3-dioxygenase. Transplant Proc 33:428-430

30. Li YP, Paczesny S, Lauret E et al (2008) Human mesenchymal stem cells license adult $\mathrm{CD} 34^{+}$hematopoietic progenitor cells to differentiate into regulatory dendritic cells through activation of the NOTCH pathway. J Immunol 1:1598-1608

31. Urban VS, Kiss J, Kovacs J et al (2008) Mesenchymal stem cells cooperate with bone marrow cells in therapy of diabetes. Stem Cells 26:244-253

32. Tritt M, Sgouroudis E, d'Hennezel E, Albanese A, Piccirillo CA (2008) Functional waning of naturally occurring $\mathrm{CD} 4^{+}$regulatory $\mathrm{T}$ cells contributes to the onset of autoimmune diabetes. Diabetes $57: 113-123$ 